\title{
A Predictive Personalised Model for the Left Atrium
}

\author{
Cesare Corrado ${ }^{1}$, Steven Williams ${ }^{1}$, Gernot Planck $^{2}$, Mark O'Neill ${ }^{1}$, Steven Niederer ${ }^{1}$ \\ ${ }^{1}$ King's College London, London, United Kingdom \\ ${ }^{2}$ Medical University of Graz, Graz, Austria
}

\begin{abstract}
We propose an integrated modelling and clinical protocol for characterising local tissue properties in the left atrium and validate the resulting personalised models in four patient cases. We generate a personalised model from a set of measured local activation times (LATs) obtained by pacing the left atrium in the proximity of the coronary sinus with a programmed pacing protocol. We validate the model by evaluating the correlation between a set of measured LATS, obtained by pacing on the high right atrium and a set numerically computed LATs. We then estimate if the tissue is capable of sustaining an atrial fibrillation or a tachycardia by triggering a spiral wave on the computational model and then analysing the activation frequencies and the time elapsed until the termination of the aberrant activation.
\end{abstract}

\section{Introduction}

Atrial fibrillation (AF) is an abnormal heart rhythm in which rapid and uncoordinated electrical activation of the atria resulting in deterioration of mechanical function. $\mathrm{AF}$ affects almost 2.5 million people in the US, [1] and is associated with an increased incidence of cardiovascular disease, stroke and premature death [2]. In drug refractory patients $\mathrm{AF}$ is treated through radio frequency catheter ablation, however, up to $40 \%$ of patients require multiple procedures. Computational models have been identified as a potential tool to help predict procedure outcomes and guide ablation targets, [3]; however, their inability to capture the significant variability in physiology typical of AF patients limits their potential to make quantitative predictions of patient response to treatment and thus to inform clinical procedures. In this work, we propose a novel method to generate and validate patient specific models of the left atria from readily available clinical measurements. In 4 patients pacing catheters were placed in the coronary sinus (CS) and the high right atrium (HRA). A 20 electrode PentaRay catheter was placed in the left atrium recording 10 bipolar electrograms (EGM). Recordings were made at up to 10 locations in each patient. At each location, an
S1-S2 pacing protocol [4] was applied at both the CS and HRA catheters to generate two conduction velocity (CV) restitution curves and an estimate of the effective refractory period (ERP) for each pair of electrodes. We fitted a modified Mitchell-Schaeffer (mMS) cardiac cell model [5] that does not exhibit pacemaker behaviour to the restitution curves recorded during CS pacing with the algorithm developed in [6]. We then interpolated the fitted cell model parameters across the atrial anatomy derived from the anatomical mapping system. Finally, we validated the model by numerically evaluating the predicted LATs obtained during simulated HRA pacing against the clinical measurements. We then triggered a spiral wave (SW) activation pattern on each of the 4 cases with a cross-field stimulus and recorded the evolution of the transmembrane potential. We identified cases of atrial fibrillation (1/4), atrial tachycardia (1/4) and self-terminating aberrant activations (2/4).

\section{Methods}

We recorded bipolar EGM during the clinical procedure and then computed LATs and evaluated the local CV restitutions and the ERP following the procedure summarised in section 2.1. We constrained the local value of the model parameters with the procedure described in section 2.2; we built a computational model with the procedure described in section 2.3. We finally validated the patient specific model as described in section 2.4.

\subsection{LATs and local CV evaluation}

We recorded local activation times by applying an external stimulus either in the proximity of CS or on the HRA and following an S1-S2 pacing protocol (3 S1=470 ms followed by a premature pacing $\mathrm{S} 2$ ). We repeated the protocol for 28 values of S2, in $2 \%$ steps and ranging between 343 and $200 \mathrm{~ms}$. We recorded bipolar EGM on the surface of the left atrium with a multipolar catheter and up to 100 electrode locations per case. For each location that an EGM was available, we evaluated LATs as the time corresponding to the first peak on the EGM trace; for each 
$\mathrm{S} 2$ we then linearly interpolated LATs values in the region covered by the catheter, and then evaluated the local CV modulus as the inverse of the modulus of the local gradient of LATs. We evaluated the local ERP as the largest S2 values that did not generate a $\mathrm{CV}$.

\subsection{Patient-specific model}

We described the local tissue electrophysiology with a heterogeneous isotropic monodomain approximation [7], and the mMS ionic model described in [5]; similarly to the original Mitchell-Schaeffer (MS) model [8], mMS is able to capture the measured $\mathrm{CV}$ and ERP restitution properties with the smallest numbers of parameters to constrain, it is proven to be stable to pacemaker behaviour independently of the choice of the parameter values and it is possible to analytically estimate the action potential duration (APD) and the $\mathrm{CV}$ restitutions with leading order approximations. We obtained locally personalised patient-specific models with the algorithm described in [6], by fitting the measured local CV restitution and the ERP to a set of pre-computed restitution curves, obtained from the solution of a $1 \mathrm{D}$ computational model on a set of known parameters. To achieve an optimal range of parameters and improve the sensitivity with respect to the measured quantities, we built the data set of pre-computed solutions by sweeping on the parameter set defined by the maximum value of $\mathrm{APD}\left(\mathrm{APD}_{\max }\right)$, the minimum value of the gate variable on the null-cline $\left(h_{\min }\right)$ defined in [5], the maximum $\mathrm{CV}\left(\mathrm{CV}_{\max }\right)$ and the $\tau_{\text {in }}$ and $\tau_{\text {open }}$ ionic parameters. We then obtained the original parameters by inverting the analytical leading order formulations. In this work, we generated a data set of 580,800 samples, evaluated with the parameter values summarised in Table 1, and keeping the gate potential value fixed and equal to $v_{\text {gate }}=0.1$.

Table 1. Parameter values used for building the data set. Each parameter spans the interval ranging from the minimum to the maximum values in increments of the step value. The set of pre-computed solutions is obtained from the Cartesian product of all of the parameter value sets.

\begin{tabular}{lccc}
\hline \hline parameter & $\min$ & $\max$ & step \\
\hline $\mathrm{CV}_{\max }(\mathrm{cm} / \mathrm{s})$ & 10 & 300 & 10 \\
$\tau_{\text {in }}(\mathrm{ms})$ & 0.01 & 0.31 & 0.03 \\
$h_{\min }$ & {$[0.01 ; 0.1]$} & {$[0.09 ; 0.5]$} & {$[0.02 ; 0.1]$} \\
$\tau_{\text {open }}(\mathrm{ms})$ & 65 & 215 & 10 \\
$\mathrm{APD}_{\max }(\mathrm{ms})$ & 120 & 270 & 15 \\
\hline \hline
\end{tabular}

\subsection{Computational model}

On the regions of the atrium where no measurements were available, we interpolated the parameter values by assigning the local values of parameters of the closest measurement point. We generated a computational mesh with an imposed edge length $\mathrm{dx}=215 \mu \mathrm{m}$ on the $2 \mathrm{D}$ surface describing the anatomy and obtained from the NavX electro-anatomical mapping system. We discretized the computational model in space with linear finite elements; we adopted a splitting technique [9] for the nonlinear term describing the ionic current. We adopted a forward-Euler scheme with a time step $\mathrm{dt}=5 \mu \mathrm{s}$ for the ionic model and a Crank-Nicholson scheme with a time step $\mathrm{dt}=50 \mu \mathrm{s}$ for the diffusive parabolic PDE. Simulations were performed with the Cardiac Arrhythmias Package (CARP), an electrophysiology solver suitable for hyper-computing [10].

\subsection{Validation process}

To validate the model we predicted LATs for each value of $\mathrm{S} 2$ recorded during the clinical procedure when pacing in HRA. In the clinical procedure the left atrium was not paced directly with the activation wave travelling to the left atrium. This means that the initial activation site on the left atrium is not known and the time it took for the activation wave to reach the left atrium needs to be subtracted from the clinically recorded LATs. The initial activation was modelled by applying an external stimulus of intensity $I_{\text {app }}=4 \mathrm{~ms}^{-1}$ and duration $t_{\text {stim }}=0.6 \mathrm{~ms}$ on a circular region with radius $\mathrm{R}=1 \mathrm{~cm}$. A three step procedure was used to estimate the centre of the stimulation. First, we interpolated the $\mathrm{CV}$ measured at the largest coupling interval S2 across the whole left atrium. Secondly, we considered each point of the mesh obtained from the electroanatomical mapping system as a potential stimulation site. For each point in the mesh we performed an eikonal activation simulation, where the activation was initialized by the candidate point, this gave a set of LATs across the whole atrium for each point in the mesh. Thirdly, we compared the predicted LATs with the measured LATs to rank each potential stimulus site and chose the point that produced the smallest error. To account for the time taken for the activation wave to reach the left atria from the pacing electrode we subtracted the mean difference between the measured and the computed LATs form the clinical LAT's prior to comparing them to the model predictions.

We summarised the predictive performance of the computational model through the following 4 quantities: the linear regression coefficients $(\mathrm{m}, \mathrm{q})$ of the regression line $y=m x+q$, where points $(x, y)$ corresponding to the measured and computed LATs for all S2 values; the coefficient of determination $r$ between measured and computed LATs; the ratio $s l$ between the two principal components of the 
covariance.

We then triggered a SW activation pattern on each of the personalised atrial models by applying a cross-field stimulus and measured the activation frequencies and the time elapsed until the termination of the aberrant activation. We applied the first stimulus at the time $t=0$ on the nodes of the lateral half of the atrium; we applied the second stimulus at time $\mathrm{t}=\mathrm{S} 2$ on the nodes of the anterior half of the atrium. We tested several values of S2 and chose those who triggered a SW lasting for at least 5 seconds.

\section{Results}

We validated the proposed method on clinical 4 cases suffering from paroxysmal atrial fibrillation (PAF) who underwent pulmonary veins isolation. Figure 1 shows the scatter plot of the measured and computed LATs for each case, while Table 2 summarises the quantities used to measure the predictive performances of the patient specific model.

Table 2. Quantities used to measure the predictive performances for each clinical case.

\begin{tabular}{ccccc}
\hline \hline Case & $\mathrm{m}$ & $\mathrm{q}$ & $r$ & $s l$ \\
\hline 1 & 0.85 & 17.88 & 0.66 & 0.19 \\
2 & 0.84 & 21.36 & 0.73 & 0.15 \\
3 & 0.68 & 36.44 & 0.73 & 0.15 \\
4 & 0.99 & 1.74 & 0.89 & 0.04 \\
\hline \hline
\end{tabular}

We then applied a cross-field stimulus on each patientspecific model with the values of S2 reported in Table 3, analysed the ability of the model to sustain an aberrant activation pattern and determined the spatial distribution of the activation frequency and the time SW self-terminated.

Table 3. Values of S2 used to trigger AF when applying a cross-field stimulation.

\begin{tabular}{lcccc}
\hline \hline & Case 1 & Case 2 & Case 3 & Case 4 \\
\hline S2 & $255 \mathrm{~ms}$ & $280 \mathrm{~ms}$ & $270 \mathrm{~ms}$ & $230 \mathrm{~ms}$ \\
\hline \hline
\end{tabular}

Cases 2 and 3 showed self-terminating SW, terminated after $\approx 16 \mathrm{~s}$ and $\approx 3.1 \mathrm{~s}$ respectively; Case 1 showed a self-sustaining AF, driven by a principal frequency of $\approx 5.64 \mathrm{~Hz}$ and a secondary frequency of $\approx 9 \mathrm{~Hz}$, characterising a localised stationary rotor. Case 4 showed an atrial tachycardia (AT), driven by a principal frequency of $\approx 4.8 \mathrm{~Hz}$. Figure 2 shows the spatial distribution of the activation frequencies after the $\mathrm{SW}$ was triggered. All the 4 models showed a dominant frequency between 4 and 6 $\mathrm{Hz}$.
Table 4 summarizes the spatial mean $\mu$ of activation frequencies, the spatial standard deviation $\sigma$ of the activation frequencies and the time $t_{\text {end }}$ elapsed before AF selfterminated.

Table 4. Spatial distribution of the activation frequencies during $\mathrm{AF}$ and time values $t_{\text {end }} \mathrm{AF}$ terminated for each of the 4 cases.

\begin{tabular}{ccccc}
\hline \hline & Case 1 & Case 2 & Case 3 & Case 4 \\
\hline$\mu[\mathrm{Hz}]$ & 5.65 & 4.11 & 4.38 & 4.8 \\
$\sigma[\mathrm{Hz}]$ & 1.28 & 0.30 & 0.32 & 0.09 \\
$t_{\text {end }}[\mathrm{s}]$ & $\infty$ & 16 & 3.1 & $\infty$ \\
\hline \hline
\end{tabular}

\section{Discussion}

Differently from [6], in this work we described the electrophysiology with the mMS model that is stable to pacemaker behaviour and was proven to furnish equivalent properties to the standard MS model as far as the SW characterising the AF is concerned, [11]. This choice improves the time performances of the algorithm since any test on the pacemaker stability on the set of fitted parameters is no longer required. Data assimilation techniques based on Kalman Filtering [12] are computationally expensive since they require solving direct problems to generate the sensitivity matrix, that is non-sparse and have a size proportional to the number of parameters to constrain. This yields subdividing the organ into regions characterised by uniform properties, limiting the degree of heterogeneity and thus the resolution. Moreover, the computational demanding related to the involved direct numerical simulations hampers the application of Kalman Filtering to a clinical framework. The method proposed in [6] conversely, allows determining the parameters characterising the tissue properties on a myocardial region that does not depends on any pre-determined subdivision, resulting in a higher resolution of the tissue heterogeneity. The same method neither requires computing the solution of a direct problem on an organ scale since it realises on a local fitting procedure against a set of pre-computed solution and thus can be applied in a clinical time scale. The simulations show how models could be used to predict if atria are capable of sustaining an arrhythmia and the type of arrhythmia that the atria can sustain. The dominant cycle lengths of the arrhythmias shown in Figure 2 are consistent with those previously reported in [13]. The models show, specifically case 1 , the potential for electrophysiological heterogeneity to be important for explaining the underlying drivers of AF. New catheters and mapping systems that are increasingly able to capture high density activation maps will provide further data to develop patient specific models that capture 

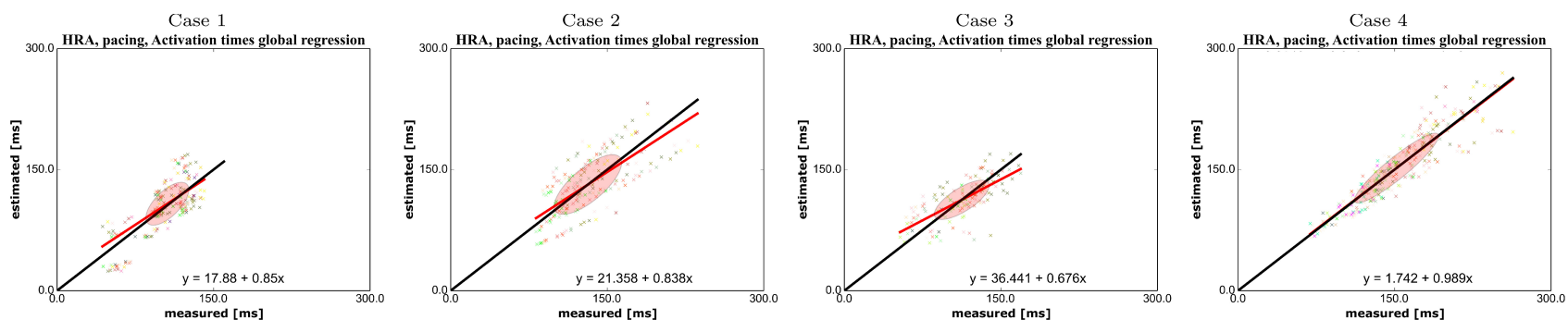

Figure 1. Scatter plot of the estimated (y-axis) vs measured (x-axis) LATs; each colour corresponds to a different S2 coupling interval. The linear regression line $y=m x+q$ is plotted in red, the line reference $y=x$ in black. The red ellipse corresponds to the covariance ellipsoid of $\mathrm{x}$ and $\mathrm{y}$.
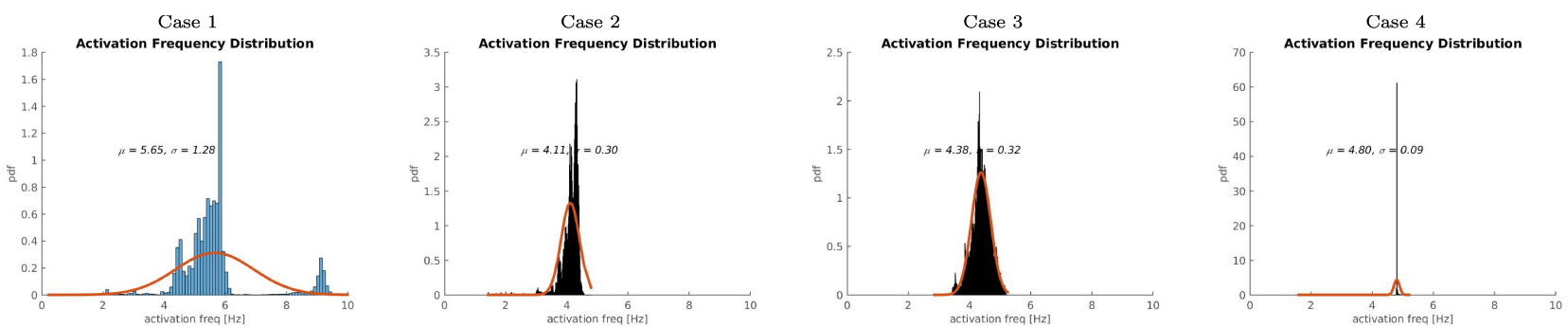

Figure 2. Space distribution of the activation frequencies for each of the 4 clinical cases

patient electrical heterogeneity.

\section{Conclusions}

We have developed an integrated modelling and clinical protocol for characterising the local tissue properties in the left atrium and validated the resulting personalised models in four patient cases.

\section{References}

[1] Colilla S, et al Estimates of current and future incidence and prevalence of the atrial fibrillation in the US adult population. Am. J. Cardiol 2013; 112:1142-1147.

[2] Camm AJ, et al Guidelines for the management of atrial fibrillation. European Heart Journal 2010; 31:2369-2429.

[3] Colli Franzone P, Pavarino L, Savarè G. Computational electrocardiology: mathematical and numerical modelling. Complex systems in Biomedicine Springler, 2006:187-241.

[4] Murgatroyd FD. Handbook of cardiac electrophysiology: a practical guide to invasive EP studies and catheter ablation. Remedica, 2002.

[5] Corrado C, Niederer S. A two-variable model robust to pacemaker behaviour for the dynamics of the cardiac action potential. Mathematical Biosciences 2016; 281:46-54.

[6] Corrado C, et al. Personalized Models of Human Atrial Electrophysiology Derived From Endocardial Electrograms. IEEE Transactions on Biomedical Engineering 2016; 64:735-742.
[7] Keener JP, Sneyd J. Mathematical physiology Springler, 1998.

[8] Mitchell C, Schaeffer DG. A Two-Current Model for the Dynamics of Cardiac Membrane. Bull Math Bio 2003; 65:767-793.

[9] Pathmanathan P, et al. Computational modelling of cardiac electrophysiology: explanation of the variability of results from different numerical solvers. Int. j. for num. meth. in biomed.ical eng. 2012; 28:890-903.

[10] Niederer S, Mitchell M, Smith N, Plank G. Simulating human cardiac electrophysiology on clinical time-scales. Front Physiol 2011; 2:14.

[11] Corrado C, et al. Predicting Spiral Wave Stability by Personalized Electrophysiology Models. In: Murray A. Computing in Cardiology. Vancouver: IEEE, 2016:229-232.

[12] Corrado C, Gerbeau JF, Moireau P. Identification of weakly coupled multiphysics problems. Application to the inverse problem of electrocardiography. J. Comp. Phys 2015; 283:271-298.

[13] Sanders P, et al. Spectral Analysis Identifies Sites of HighFrequency Activity Maintaining Atrial Fibrillation in $\mathrm{Hu}-$ mans. Circulation 2005; 112:789-797.

Address for correspondence:

Cesare Corrado

Dep. of Biomedical Engineering; St Thomas' Hospital, SE17EH, London (UK)

cesare.corrado@kcl.ac.uk 